\title{
Comparison of Analysis Routines for EDS and EELS Spectrum Images of Electrical Contacts to Single-Walled Carbon Nanotubes.
}

\author{
Joshua D. Sugar ${ }^{1}$, Alexander A. Kane ${ }^{1}$, Alexandra C. Ford ${ }^{1}$, Michael J. Rye ${ }^{2}$, Lisa M. Lowery ${ }^{2}$, and \\ François Léonard ${ }^{1}$ \\ 1. Sandia National Laboratories, Livermore, CA, USA. \\ 2. Sandia National Laboratories, Albuquerque, NM, USA.
}

Carbon nanotube (CNT) devices offer potential alternatives to traditional CMOS FETs. They have several advantages because of their ultrathin dimensions and ballistic charge carrier transport [1-3]. To have efficient electrical transport of charge carriers into CNT channels, it is important that the metal and the carbon nanotube be in intimate contact, with no barriers to charge transport. Because a typical semiconducting single-walled CNT has a diameter of approximately $1 \mathrm{~nm}$ and CNTs are usually embedded in metal to form good electrical contacts, analytical TEM is required to study the morphology and chemistry of that interface. Here, we use EDS and EELS to study the CNT/electrical contact interface, and make connections between solution-based processing steps and the resulting contact structure. These results can eventually be used to optimize fabrication parameters.

An important question is whether the metal contact forms a conformal layer around the CNT because interfacial morphology affects the contact area and resistance. While other studies have also looked at $\mathrm{CNT} /$ contact morphology [4-7], this structure is very sensitive to the device fabrication parameters. Therefore, it is necessary to investigate the contact structure for each set of fabrication conditions. Because we use solution-processed CNTs, it is possible for surfactants and other solvents to leave behind residues that contaminate the interface. Here, we use a probe-corrected FEI G2 80-200 with ChemiSTEM and a Gatan Quantum 963 to perform EELS and EDS on cross sections of CNT contacts.

Figure 1 shows an example of an EELS spectrum image for a CNT contact. In figure 1, a conventional windowed mapping approach is used to identify the spatial distribution of the elements. By comparison, figure 2 analyzes the same data using multivariate statistical analysis (MSA) [8]. In this example, the MSA reveals information in the $\mathrm{O}$ pre-peak that is difficult to determine from conventional mapping alone. Additional, the morphology of the $\mathrm{SiO}_{2}$ around the bottom of the CNT is more easily visualized with MSA. A more rapid understanding of the interfacial chemistry near the contact is gained by combining a variety of algorithms to analyze the data. We will compare several useful strategies that can aid in data analysis.

[1] A.D. Franklin and Z. Chen, Nat Nano 5 (2010)

[2] A. Javey, et al., Nature 424 (2003)

[3] L. Wei, et al., Ieee Transactions on Electron Devices 58 (2011)

[4] A.D. Franklin, et al., Nano Letters 13 (2013)

[5] A.D. Franklin, et al., Nano Letters 12 (2012)

[6] A. Javey, et al., Nature Materials 1 (2002)

[7] D. Shahrjerdi, et al., ACS Nano (2013)

[8] P.G. Kotula and M.R. Keenan, Microscopy and Microanalysis 12 (2006)

[9] H. Kurata, et al., Physical Review B 47 (1993)

[10] Sandia National Laboratories is a multi-program laboratory managed and operated by Sandia Corporation, a wholly owned subsidiary of Lockheed Martin Corporation, for the U.S. Department of Energy's National Nuclear Security Administration under contract DE-AC04-94AL85000. 


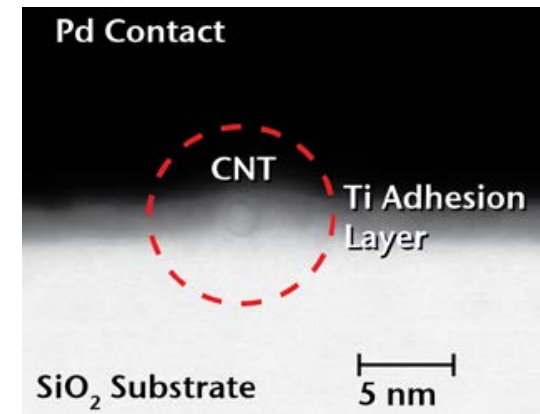

(a)

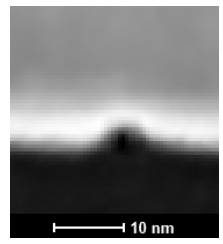

(d)

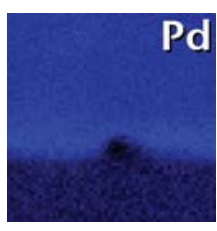

(e)

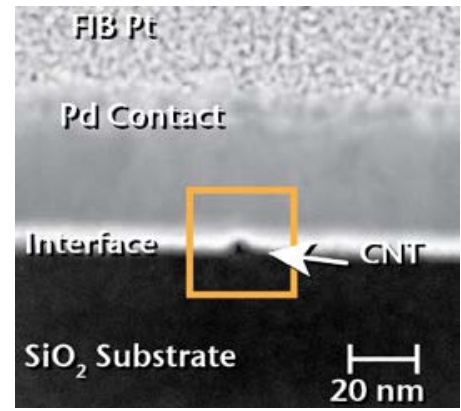

(b)

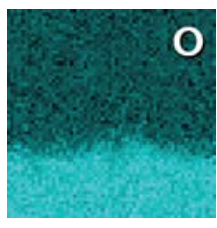

(f)

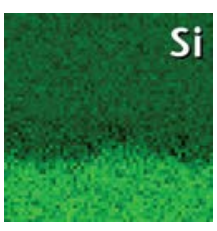

(g)

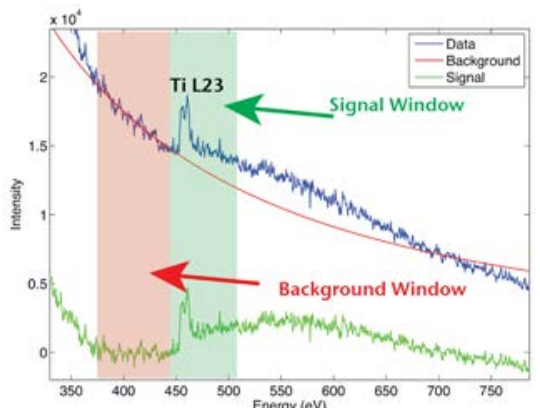

(c)

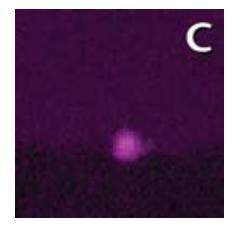

(h)

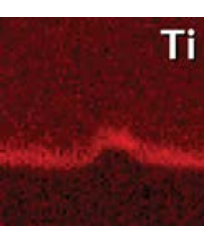

(i)

Figure 1. (a) BF STEM showing a typical cross section of a CNT contact. The CNT is visible in the image. In (b), a HAADF STEM image of a different contact is shown and the region selected for spectrum imaging is shown in the orange box. An illustration of the conventional windowed mapping technique is shown in (c) for the Ti L23 edge and implemented in (e) - (i) for $\mathrm{Pd}, \mathrm{O}, \mathrm{Si}, \mathrm{C}$, and Ti, respectively. The HAADF image in (d) shows the scale of the maps in (e) - (i) at $0.5 \mathrm{~nm} /$ pixel resolution.

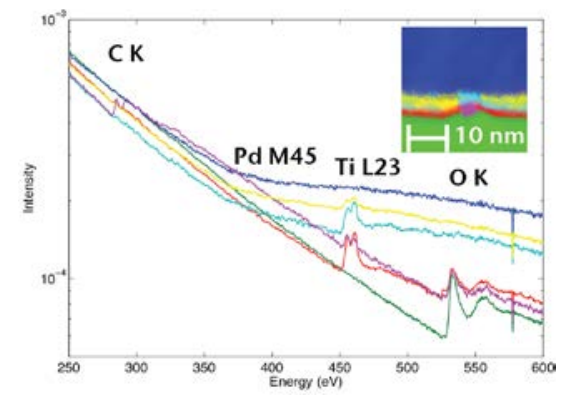

(a)

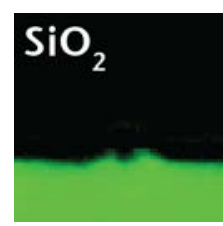

(c)

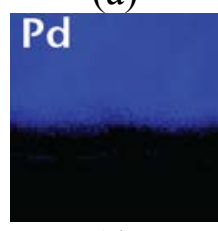

(d)

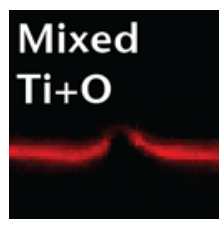

(e)

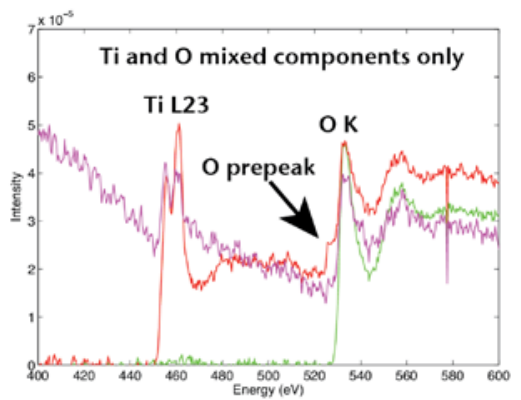

(b)

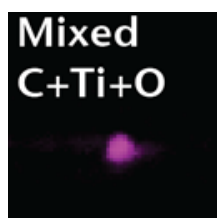

(f)

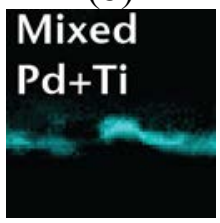

(g)

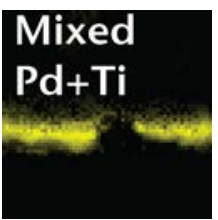

(h)

Figure 2. MSA analysis is performed on the same data as Figure 1. A composite image showing the spatial distribution of each "pure" component and its corresponding spectrum that results from a multivariate statistical analysis routine are shown in (a). Each of the 6 "pure" components is shown in (c) - (h). A close inspection of the mixed $\mathrm{Ti}$ and $\mathrm{O}$ components in (b) shows that the red component has intensity in the position of the $\mathrm{O}$ prepeak, indicative of hybridized O $2 \mathrm{p}$-Ti $3 \mathrm{~d}$ vacant orbitals [9]. This feature might have been missed if conventional mapping were the only analysis performed. 